\title{
Farmacologica Follia (pensieri di una medicina nelle vene di un paziente)
}

\section{Leonardo Mari}

Farmacista Dirigente I livello S.O.S., Farmacia Ospedale Mugello, Azienda USL Toscana Centro, Firenze

\section{Pharmacological folly (thoughts of a drug in the veins of a patient)}

A fantasy about a drug being the narrator of a story about the added well-being a patient gains by telling what's on his mind.

Keywords: Efficacy, Narration, Necessity, Optimism, Simplicity

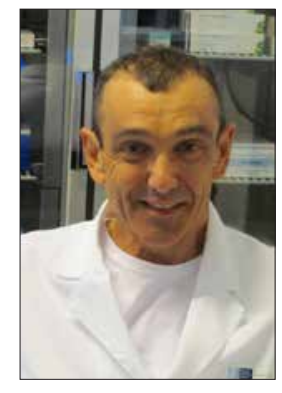

Leonardo Mari ...eppure qualcuno dovrebbe aver capito! Ormai sono anni!

Sono anni che noi molecole di farmaci diciamo che il nostro lavoro potrebbe essere più facile.

Oggi il mio paziente era un po' triste, molto triste, tanto che quasi mi avrebbe rifiutata. Poi, seduto sulla panchina dei giardini ha visto quei bambini giocare e $\mathrm{i}$ suoi occhi si sono illuminati!

Ohh..! Come avrebbe voluto parlare con qualcuno della sua infanzia!

Ve lo dico perché son destinata a fermarmi nei suoi neuroni e da lì si sente tutto, non si scappa, le bugie non sono ammesse.

\section{Piezze 'e core}

Ti amo bimbo mio per te voglio tutto il bene.

Voglio ascoltare i tuoi perché e quel che non sai dirmi.

Accepted: May 6, 2017

Published online: June 5, 2017

Indirizzo per la corrispondenza:

Dr. Leonardo Mari

ASL 10 (FI) Farmacia Ospedale Mugello

V.le Resistenza

50032 Borgo San Lorenzo (FI)

leonardo.mari@uslcentro.toscana.it

\author{
Ecco, qui ed ora \\ quanto di più grande \\ posso darti \\ è la consapevolezza di me stesso.
}

Questo pensava! Pensava ai suoi ricordi di bambino, alle esperienze di genitore e poi di nonno.

Pensava che domani, disteso sul suo letto in ospedale, avrebbe raccontato tutto all'infermiera.

Lei è un po' burbera ma gli è sempre sembrata una brava persona e valeva la pena che fosse lui a fare le prime vere confidenze.

Forse poi, parlando, tutto poteva essere diverso e forse si sarebbe sentito meglio e meno malato e tutto avrebbe avuto un altro senso: la dialisi, la terapia, il tempo passato attaccato alla macchina.

Sì! Voleva fuggire da quei brutti pensieri che lo assalivano ogni tanto.

Spente le luci

$$
\begin{gathered}
\text { Siamo soli. Anche d'amore } \\
\text { avvolti in un abbraccio } \\
\text { soli, sentiamo scorrere piano } \\
\text { nel letto delle vene } \\
\text { un mare grande; } \\
\text { e ci spaventa il rombo } \\
\text { la calma e la maestà dell'onda } \\
\text { padre e madre d'una vita } \\
\text { che ha solo una certezza. }
\end{gathered}
$$

Ecco, sì!.. Sono questi i suoi brutti pensieri e forse..., come mi hanno detto le mie cugine medicine, non son diversi da quelli di tanti altri. 
Oggi però, credo che il mio paziente abbia davvero acceso l'ottimismo che c'è in lui!

Ha parlato con l'infermiera dei suoi ricordi e si è sentito bene quando lei lo ascoltava.

Lei non ha mai detto molte cose, non lo ha mai consigliato, né tanto meno accusato, lo ha solo ascoltato e questo bastava per farlo stare meglio.

Mentre parlava e si confidava aveva l'impressione che tutto fosse più facile e più semplice.

Ecco, sì! Semplice, questo è davvero importante. Sono le cose semplici quelle che funzionano meglio.

\section{Semplicemente}

Di stridule voci, la noia fiacca il pensiero come l'afa d'agosto. Mani incerte spingono inutili, pesanti macigni su per la salita.

Nel grigio di questo mercato

lo sguardo s'affascina nell'oro del pane, nella grazia di una stilla d'acqua.

Un cibo semplice: questo reclama ogni battito, ogni respiro, ogni sogno spezzato. 